\title{
Application of cerebral oximetry for a parturient with Takayasu's arteritis undergoing cesarean section
} -a case report-

\author{
Eun Hye Lee, Eunsu Choi, and Wonsik Ahn \\ Department of Anesthesiology and Pain Medicine, Seoul National University College of Medicine, Seoul, Korea
}

Takayasu's arteritis (TA) is a chronic inflammatory disease involving the aorta. Because TA sometimes involves cerebral arteries, anesthetic debates focus on cerebral monitoring. There is limited evidence as to which cerebral monitoring method is most adequate. Furthermore, there is insufficient evidence to determine which anesthetic technique is better for TA parturients. We experienced the case of a TA parturient who developed transient cerebral ischemia during cesarean section. The patient's TA involved her cerebral arteries, and her regional cerebral oxygen saturation $\left(\mathrm{rSO}_{2}\right)$ was lower in the left side than in the right side. She complained of speech impairment, tinnitus, and stiffness of the posterior neck when the $\mathrm{rSO}_{2}$ levels dropped. The FloTrac/Vigileo ${ }^{\mathrm{TM}}$ system did not correlate with clinical symptoms, but the cerebral oximeter displayed the low oxygen saturation. We recommend the cerebral oximetry for cerebral monitoring in TA parturients who undergo cesarean sections, especially in hemodynamically unstable patients under regional anesthesia or unconscious patients under general anesthesia. (Korean J Anesthesiol 2013; 65: 158-162)

Key Words: Cerebral oximetry, Cesarean section, Epidural anesthesia, Near-infrared spectroscopy, Takayasu's arteritis.

Takayasu's arteritis (TA) is a rare idiopathic inflammatory disease that is characterized by progressive obliteration of the aorta and its large branches which causes stenosis, occlusion and aneurysms of systemic and pulmonary arteries [1]. TA is a chronic vasculitis that primarily affects relatively young age women, usually between 10 and 40 years [2]. Thus, the safety of pregnancy should be considered in women of reproductive age with TA. Anesthesia for a TA parturient is complicated by aneurysmal ruptures, cerebral ischemia and uncontrolled hypertension. Since TA is manifested by aortic aneurysms or stenosis of cerebral arteries, increases in blood pressure can cause aneurysmal ruptures and decreases in blood pressure which lead to cerebral ischemia.

Blood flow to the fetus as well as to the major organs of the parturient must be maintained during anesthesia for cesarean sections. Special attention must be paid to maintaining cerebral blood flow and to protecting cerebral ischemia during anesthesia because cerebral vascular insufficiency is common in a TA

Received: June 15, 2012. Revised: 1st, August 17, 2012; 2nd, September 9, 2012; 3rd, September 10, 2012. Accepted: September 20, 2012.

Corresponding author: Wonsik Ahn, M.D., Department of Anesthesiology and Pain Medicine, Seoul National University College of Medicine, 101, Daehak-ro, Jongno-gu, Seoul 110-744, Korea. Tel: 82-2-2072-3087, Fax: 82-2-766-3087, E-mail: aws@snu.ac.kr

(c)This is an open-access article distributed under the terms of the Creative Commons Attribution Non-Commercial License (http:// creativecommons.org/licenses/by-nc/3.0/), which permits unrestricted non-commercial use, distribution, and reproduction in any medium, provided the original work is properly cited. 
patient as this disease often involves arteries supplying cerebral blood flow. Although maintenance of systemic blood pressure is critical in TA patients, there have been few reports concerning intraoperative cerebral monitoring. This case presents a TA parturient who was monitored by cerebral oximetry for the prevention of cerebral hypoperfusion during cesarean section.

We recommend cerebral oximetry for cerebral monitoring in TA parturients who are undergoing cesarean sections, especially in hemodynamically unstable TA parturients under regional anesthesia or who are unable to express cerebral symptoms under general anesthesia.

\section{Case Report}

A $53 \mathrm{~kg}, 157 \mathrm{~cm}, 35$-year-old primigravida at the 38th week of pregnancy was admitted for a cesarean section due to breech presentation. She had been diagnosed with TA 7 years prior to this presentation. However she refused medications and further evaluation until delivery. Computed tomographic angiography performed 3 years prior had revealed that the left common carotid artery and left subclavian artery were totally occluded along with a thrombotic aneurysm $5.8 \times 5.2 \times 9.6 \mathrm{~cm}$ in size (Fig. 1). She also had a localized dissection in the low abdominal aorta and focal stenosis in the descending aorta as well as a large left coronary aneurysm and about 50\% stenosis of the right coronary artery. Plain chest radiographs revealed a huge superior mediastinal mass which was suspected of an aneurysm with thrombus in the left subclavian artery (Fig. 2).

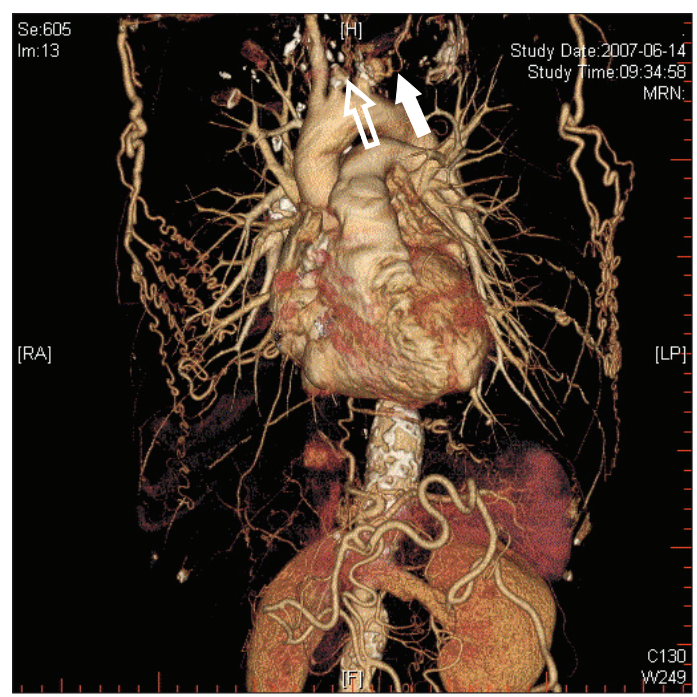

Fig. 1. Three-dimensional computed tomographic angiography for the diagnostic evaluation of Takayasu's arteritis performed 3 years prior to admission. It revealed total occlusion of the left common carotid (open arrow) and left subclavian arteries (closed arrow) along with a thrombotic aneurysm measuring $5.8 \times 5.2 \times 9.6 \mathrm{~cm}$.
Cardiac single photon emission computed tomography and echocardiography were performed preoperatively to follow up on her coronary artery statuses. These examinations showed an old myocardial infarct, a persistent perfusion defect in the basal inferior wall and a reduction in wall motion.

The patient had been diagnosed with Type III TA as determined by the involvement of the aortic arch and coronary artery as well as the descending thoracic and abdominal aortas. She developed dyspnea on exertion with New York Heart Association Functional Class III. On physical examination, peripheral pulsations were weak in all limbs except the right arm. Heart rate was 120 beats/min, and non-invasive blood pressure (NIBP) was $96 / 78 \mathrm{mmHg}$ in the right upper limb and 95/69 $\mathrm{mmHg}$ in the left upper limb. NIBP in the lower limbs measured 148/99 $\mathrm{mmHg}$ on the right and 156/95 $\mathrm{mmHg}$ on the left. She was cooperative and neurological examinations indicated normal results. A slowly titrated epidural anesthesia was selected to maintain hemodynamic stability. Regional anesthesia functioned as a simple and reliable monitor of cerebral perfusion through the patient's level of consciousness. For these reasons, we planned and executed an elective caesarean section under epidural anesthesia.

On arrival at the operating room, we began intraoperative monitoring by electrocardiography, pulse oximetry and NIBP measurement. Electrocardiography was unremarkable with a heart rate of 126 beats/min and the pulse oximeter on her right index finger displayed a normal waveform with a saturation of 95-96\% in room air. She was alert and oriented, and her initial NIBP was 113/77 mmHg in the right upper limb. Her left radial, brachial and axillary arteries pulsed feebly, whereas her brachial and axillary arterial pulsations were stronger on the right than

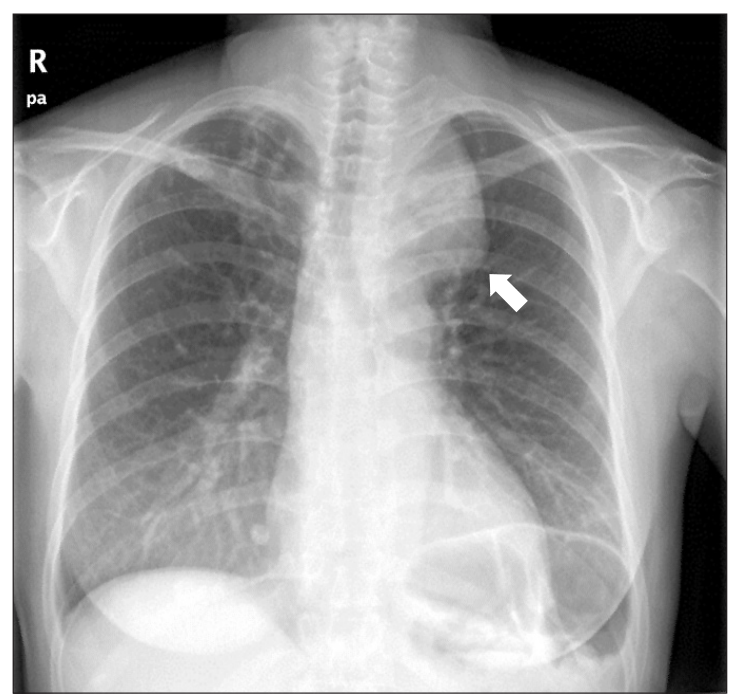

Fig. 2. An aneurysm with thrombus in the left subclavian artery (arrow) showing a huge superior mediastinal mass in a plain chest radiograph. 


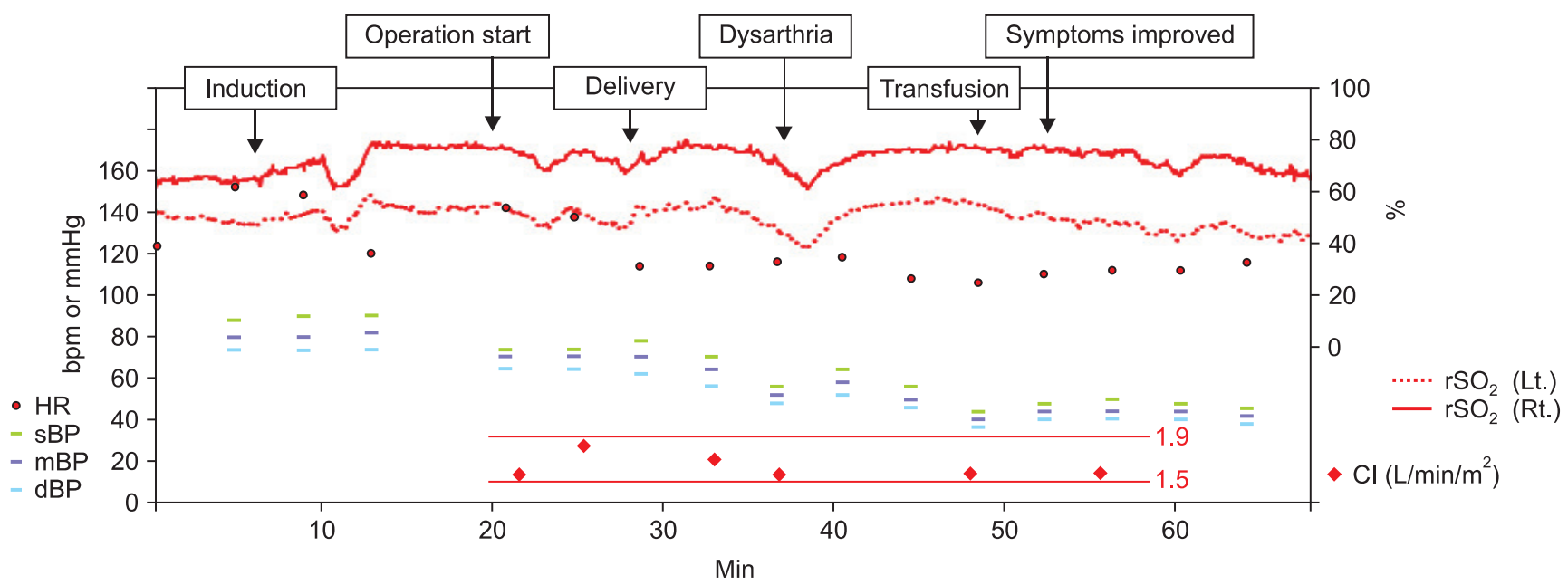

Fig. 3. Intraoperative changes in regional cerebral oxygen saturation, heart rate, blood pressure and cardiac output during cesarean section. The rSO ${ }_{2}$ value dropped 6 minutes after delivery and was accompanied by neurologic symptoms, including dysarthria. The neurologic symptoms improved as $\mathrm{rSO}_{2}$ increased, although arterial blood pressure and cardiac index did not correlate with the symptoms $\left(\mathrm{rSO}_{2}\right.$ : regional cerebral oxygen saturation, HR: heart rate, sBP: systolic arterial blood pressure, dBP: diastolic arterial blood pressure, mBP: mean arterial blood pressure, CI: cardiac index; bpm, beats per minute).

on the left. An artery cannula was placed in the right radial artery under local anesthesia connected to a FloTrac ${ }^{\mathrm{TM}}$ sensor and Vigileo ${ }^{\mathrm{TM}}$ monitor system (Edward Lifesciences, Irvine, CA, USA) device for continuous cardiac output assessments to monitor continuous blood flow. The initial cardiac index was $1.5 \mathrm{~L} / \mathrm{min} / \mathrm{m}^{2}$. A cerebral oximeter probe (Invos ${ }^{\mathrm{TM}}$, Cerebral/ Somatic Oximeter 5100C, Somanetics Corporation, Troy, MI, USA) was attached to the forehead. The initial regional cerebral oxygen saturation $\left(\mathrm{rSO}_{2}\right)$ levels were $64 \%$ on the right and $51 \%$ on the left.

After placing the patient in the left lateral recumbent position, the median approach was performed with loss of resistance technique using an $18 \mathrm{G}$ Tuohy needle between the L4 and L5 lumbar spines. The catheter was inserted and fixed. Her position was then changed to the supine position with a wedge under her right hip. The height of the block was increased gradually to T5 by using $11 \mathrm{ml}$ of $2 \%$ lidocaine injected through an epidural catheter. Oxygen was administered via a Venti ${ }^{\mathrm{R}}$ mask, and a capnometer was installed below her nose to monitor respiration. The value of $\mathrm{rSO}_{2}$ was stable. Ten minutes after skin incision, a 3,180 gram girl was delivered and her Apgar score was 8 at 1 minute and 9 at 5 minutes.

Three minutes after delivery, the placenta was removed and $20 \mathrm{IU}$ of oxytocin diluted in $1,000 \mathrm{ml}$ of $0.9 \%$ saline continued to be intravenously infused. The $\mathrm{rSO}_{2}$ levels dropped from 64 to $61 \%$ on the right and from 51 to $39 \%$ in the left at 6 minutes after delivery. At that time, we were able to obtain NIBP measurements. Arterial blood pressure was 56/48 $\mathrm{mmHg}$ without changes in cardiac index. She complained of dysarthria, tinnitus, and stiffness of the posterior neck (Fig. 3).

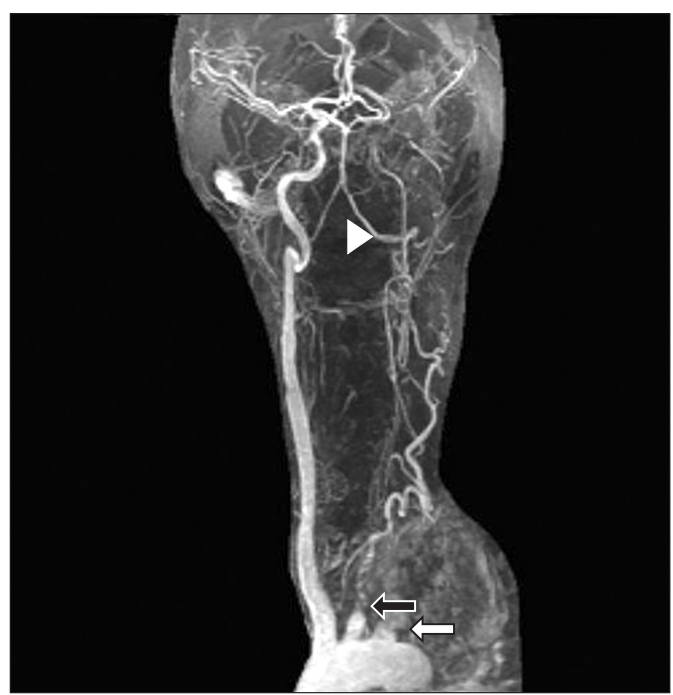

Fig. 4. Magnetic resonance angiography showing a complete occlusion of the left common carotid (black arrow) and subclavian arteries (white arrow). There was a near complete occlusion of bilateral proximal vertebral arteries. The left distal vertebral artery (arrow head) was reconstituted by the left thyrocervical trunk.

An intravenous infusion of phenylephrine $(100 \mathrm{mcg})$ and rapid administration of colloid ( $300 \mathrm{ml}$ over 10 minutes) improved dysarthria, tinnitus and stiffness of the posterior neck as $\mathrm{rSO}_{2}$ increased from 61 to $78 \%$ on the right and from 39 to $56 \%$ on the left. There was no corresponding change in arterial blood pressure and cardiac index with the increase of the NIBP to $81 / 71 \mathrm{mmHg}$. However, she still complained of numbness of the face and blurred vision. Her laboratorial test results were as 
follows: hemoglobin, $9.0 \mathrm{mg} / \mathrm{dl}$; hematocrit, $26 \%$; $\mathrm{Na}^{+}, 135 \mathrm{mg} / \mathrm{dl}$; $\mathrm{K}^{+}, 3.03 \mathrm{mg} / \mathrm{dl}$; and $\mathrm{Ca}^{2+}, 0.85 \mathrm{mmol} / \mathrm{L}$.

The patient's facial numbness and blurred vision were resolved 20 minutes after the neurologic symptoms developed. Over time, her arterial compliance was reduced and invasive arterial blood pressure waveform damped along with a progressive decline in pulse pressure (Fig. 3 ). She lost about 1,000 $\mathrm{ml}$ of blood and received, in total, $500 \mathrm{ml}$ of colloid solution, 2,500 $\mathrm{ml}$ of crystalloid, and 1 unit of blood were infused.

The patient was transferred to the intensive care unit for special attention to neurologic symptoms with continuous arterial blood pressure monitoring. Even though no focal neurologic deficits, postoperative brain magnetic resonance imaging and a magnetic resonance angiographic study were performed to rule out acute cerebral infarction. Magnetic resonance imaging showed multiple old infarcts in the right frontal and left frontotemporoparietal areas and a suspicious acute left cerebellar small infarct. In addition, there were complete occlusions of the left subclavian, left common carotid, internal carotid and middle cerebral arteries. There were near-complete occlusions of the bilateral proximal vertebral arteries. The left distal vertebral artery was reconstituted by the left thyrocervical trunk (Fig. 4). She refused further treatments, including surgery. She was discharged on the sixth postoperative day.

\section{Discussion}

Although TA occurs primarily in young women of childbearing age, there is little information on the effect of pregnancy and its management in such women. Perioperative management of delivery in TA patients is challenging for anesthesiologists. Because the disease often involves arteries supplying cerebral blood flow, the maintenance of cerebral blood flow and the protection of cerebral ischemia during anesthesia are essential. Nevertheless, there is insufficient evidence for optimal anesthetic guidelines for the prevention of cerebral hypoperfusion. There is still limited evidence of the potential benefits of cerebral monitoring in detecting cerebral ischemia [3]. In our case, a cesarean section was carried out in a parturient with severe TA under epidural anesthesia with cerebral monitoring using transcranial cerebral oximetry. Changes in $\mathrm{rSO}_{2}$ are useful for monitoring and detecting cerebral hypoxia events resulting from hemodynamic compromise. Therefore, cerebral oximetry can be used for neurological monitoring in hemodynamically unstable patients under regional anesthesia or unconscious patients under general anesthesia.

TA was first described in 1908 and named in 1942 by a Japanese ophthalmologist, Takayasu, who observed retinopathy in the absence of peripheral pulses [2]. TA is a granulomatous vasculitis of the aorta and its major branches. Four types of
TA can be identified [4]. Type I involves the aortic arch and its main branches. Type II is restricted to the descending thoracic and abdominal aortas. Type III shows features of both Types I and II. Type IV additionally involves the pulmonary artery. Coronary artery involvement is an even more rare condition and is detected in approximately 10 to $30 \%$ of all cases. The extent and severity of the specific arteries involved determine clinical features [5]. Although the causes of TA are unknown, they include autoimmunity, gender hormones (more common in young females), and genetic factors (as demonstrated by the predisposition of the human leukocyte antigen - HLA BW52) [2].

Pregnancy does not interfere with the progression and outcome of TA [6]. However, circulation may be impaired and hypertension may be aggravated by the increased intravascular volume during pregnancy. Severe uncontrolled hypertension and its effect on the heart, kidney and brain are a major cause of morbidity and mortality in TA parturients. Clinical manifestations related to hypertension are congestive heart failure, progression of renal insufficiency and hemorrhagic complications.

It is important to prevent hypotension in order to avoid hypoperfusion of major organs and the fetus. From this point of view, prevention of cerebral hypoperfusion is also important and cerebral function monitoring is necessary. Regional anesthesia is preferred because it allows for monitoring of cerebral perfusion through the patient's level of consciousness. Since consciousness can be used as a simple and reliable monitor of neurological functions, it grants direct monitoring of brain activities. Furthermore, regional anesthesia using a slowly titrated level of neuraxial blockade may easily and safely prevent large swing in blood pressure [7]. Epidural anesthesia with slow installation of the blockade causes hypotension less frequently and requires fewer vasopressors than a spinal blockade. Hemodynamic stability is preferably maintained by the levels of a slowly titrated blockade. Therefore, we chose slowly titrated epidural blockade.

In this case, we used the FloTrac/Vigileo ${ }^{\mathrm{TM}}$ system for continuous monitoring of cardiac output. The FloTrac/Vigileo ${ }^{\text {TM }}$ system seems to correlate well with transthoracic echocardiography except for changes in circulatory status in spontaneously breathing patients, even though the system is acceptable in mechanically ventilated patients [8]. Futhermore, it is a reliable method for minimally invasive hemodynamic monitoring in parturients undergoing cesarean sections with spinal anesthesia [9]. However, regional circulation affected by stenoses of major blood vessels and reduced vascular elasticity cause complications in blood pressure monitoring of TA patients [7]. As a result, there are some potential limitations to accuracy and validity in patients with reduced arterial compliance like TA. In our case, cardiac index was within $1.5-1.9 \mathrm{~L} / \mathrm{min} / \mathrm{m}^{2}$. The value was unreliable and the difference between "actual" and recorded 
arterial BP may be exaggerated by hypotension with subsequent hemodynamic compromise. For this reason, we had to use another supplementary monitor (e.g., cerebral hemodynamic monitors: transcranial Doppler measurement, jugular venous oxygen saturation or transcranial cerebral oximetry) to assess cardiovascular function for maintenance of blood flow to major organs. Although transcranial regional cerebral oxygen saturation represents local oxygen saturation, a decrease in $\mathrm{rSO}_{2}$ correlated well with a decrease in BP under beach chair position for shoulder surgery [10]. The transcranial regional cerebral oxygen saturation is a simple but reliable index of hypotension during spinal anesthesia for cesarean sections [11].

Even though we performed regional anesthesia, we used cerebral oximetry for non-invasive monitoring of cerebral perfusion in the patient while she was awake. In our case, the initial value of $\mathrm{rSO}_{2}$ was lower in the left side than in the right side, which was suggestive of the left basal cerebral hypoperfusion due to the stenotic lesions of left common carotid, internal carotid and middle cerebral arteries. In particular, changes in $\mathrm{rSO}_{2}$ provided additional information about cerebral perfusion when conventional methods, such as peripheral oxygenation and/or systemic hemodynamics, are not be predictive [3]. These results imply that $\mathrm{rSO}_{2}$ is useful for detecting and managing cerebral ischemic events in patients with cerebrovascular insufficiencies under regional anesthesia or unconscious patients under general anesthesia. We believe that acute decreases in $\mathrm{rSO}_{2}$ are associated with hypotension in a compromised cardiovascular system. Although monitoring cerebral perfusion and metabolic changes has not yet been widely used, some studies suggest their usefulness in critically ill patients $[10,12]$.

In conclusion, anesthetic management in TA patients focuses on maintaining hemodynamic stabilities. Although pregnancy does not interfere with disease progression, it is often fatal due to cerebral ischemia or heart failure. Therefore, anesthesiologists should be aware of intensive peripartum management care [13]. The anesthetic goal in TA parturients is the maintenance of blood pressure for perfusion to major organs and the fetus during perioperative periods. Prevention of cerebral hypoperfusion is even more crucial in parturients who are diagnosed with TA, especially involving the carotid artery. Transcranial regional cerebral oxygen saturations evaluated by cerebral oximetry is a simple but reliable index in such particular cases. Our case suggests that it may be advisable to monitor cerebral perfusion by cerebral oximetry and to maintain perioperative stable hemodynamics by using slowly titrated epidural anesthesia.

\section{References}

1. Beilin Y, Bernstein H. Successful epidural anaesthesia for a patient with Takayasu's arteritis presenting for caesarean section. Can J Anaesth 1993; 40: 64-6.

2. Wilke WS. Large vessel vasculitis (giant cell arteritis, Takayasu arteritis). Baillieres Clin Rheumatol 1997; 11: 285-313.

3. Smythe PR, Samra SK. Monitors of cerebral oxygenation. Anesthesiol Clin North America 2002; 20: 293-313.

4. Rav-Acha M, Plot L, Peled N, Amital H. Coronary involvement in Takayasu's arteritis. Autoimmun Rev 2007; 6: 566-71.

5. Park YB, Hong SK, Choi KJ, Sohn DW, Oh BH, Lee MM, et al. Takayasu arteritis in Korea: clinical and angiographic features. Heart Vessels Suppl 1992; 7: 55-9.

6. Sharma BK, Jain S, Vasishta K. Outcome of pregnancy in Takayasu arteritis. Int J Cardiol 2000; 75 Suppl 1: S159-62.

7. Kathirvel S, Chavan S, Arya VK, Rehman I, Babu V, Malhotra N, et al. Anesthetic management of patients with Takayasu's arteritis: a case series and review. Anesth Analg 2001; 93: 60-5.

8. Biais M, Vidil L, Sarrabay P, Cottenceau V, Revel P, Sztark F. Changes in stroke volume induced by passive leg raising in spontaneously breathing patients: comparison between echocardiography and Vigileo/FloTrac device. Crit Care 2009; 13: R195.

9. Auler JO Jr, Torres ML, Cardoso MM, Tebaldi TC, Schmidt AP, Kondo MM, et al. Clinical evaluation of the Flotrac/Vigileo system for continuous cardiac output monitoring in patients undergoing regional anesthesia for elective cesarean section: a pilot study. Clinics (Sao Paulo) 2010; 65: 793-8.

10. Fischer GW, Torrillo TM, Weiner MM, Rosenblatt MA. The use of cerebral oximetry as a monitor of the adequacy of cerebral perfusion in a patient undergoing shoulder surgery in the beach chair position. Pain Pract 2009; 9: 304-7.

11. Berlac PA, Rasmussen YH. Perioperative cerebral near-infrared spectroscopy (NIRS) predicts maternal hypotension during elective caesarean delivery in spinal anaesthesia. Int J Obstet Anesth 2005; 14: 26-31.

12. Pugliese F, Ruberto F, Tosi A, Martelli S, Bruno K, Summonti D, et al. Regional cerebral saturation versus transcranial Doppler during carotid endarterectomy under regional anaesthesia. Eur J Anaesthesiol 2009; 26: 643-7.

13. Ioscovich A, Gislason R, Fadeev A, Grisaru-Granovsky S, Halpern S. Peripartum anesthetic management of patients with Takayasu's arteritis: case series and review. Int J Obstet Anesth 2008; 17: 358-64. 